\title{
Sensitivity Enhancement of Nonlinear Waveguide Sensors With Conducting Graphene Layer: TE Mode
}

\author{
Hala J. EL-KHOZONDAR ${ }^{1 *}$, Mohammed M. SHABAT ${ }^{2}$, and Rana KHLIFA ${ }^{2}$ \\ ${ }^{1}$ Electrical Engineering department, Islamic University of Gaza, P.O.Box 108, Gaza, Palestine \\ ${ }^{2}$ Physics department, Islamic University of Gaza, P.O.Box 108, Gaza, Palestine \\ *Corresponding author: Hala J. EL-KHOZONDAR_Ｅ-mail: hkhozondar@iugaza.edu.ps
}

\begin{abstract}
We propose a three-layer waveguide sensor. The proposed sensor consists of a graphene thin layer with constant conductivity at the interface between air and dielectric media with thickness $d$ sitting above a nonlinear layer. The sensitivity of the sensor is derived from the dispersion equation. The sensitivity is calculated for both $\mathrm{TE}_{0}$ and $\mathrm{TE}_{1}$. Results show that the sensitivity of the proposed sensor depends on the conductivity of the graphene layer, the angular frequency, and the thickness of the dielectric layer.
\end{abstract}

Keywords: Graphene; nonlinear Kerr like materials; optical sensor; waveguide sensor

Citation: Hala J. EL-KHOZONDAR, Mohammed M. SHABAT, and Rana KHLIFA, "Sensitivity Enhancement of Nonlinear Waveguide Sensors With Conducting Graphene Layer: TE Mode," Photonic Sensors, 2018, 8(1): 29-33.

\section{Introduction}

In waveguide sensors, the evanescent field can detect changes that occur in the neighboring region as a response for the change in refractive index. These sensors have various applications such as biosensing, chemical sensing, biochemical sensing, and humidity sensing [1-7]. For example, biosensor is used for biosensing, which is a process that detects the interactions of biological objects with a waveguide surface. The refractive index of the surface changes once an analytic particle fixes to the surface of the waveguide, resulting in changes in the evanescent field, which can be measured.

Previous attempts to improve the performance of sensors have been introduced [8-16]. In order to improve the performance of sensors in practical applications, the sensitivity should be maximized. Therefore, the appropriate sensor strata should be selected such that the refractive index change is extreme. The propagation of light in a media with intensity dependent refractive index is called nonlinear media. An intensively investigated nonlinear media, in which refractive index is directly proportional to the field intensity, is called Kerr-like media [11, 12, 14].

Graphene is added to the proposed waveguide structure to enhance its performance. Graphene is a novel two-dimensional crystalline material consisting of attached carbon atoms oriented in a hexagonal layer, with a carbon-carbon distance of $0.142 \mathrm{~nm}$. It has marvelous properties. The electrons in graphene behave as if they are massless due to the interaction between electrons and hexagonal lattice. Therefore, electrons in graphene are governed by Dirac equation and named Dirac fermions [17]. Chen et al. reported that graphene conductance depended on frequency and resistivity became

Received: 11 August 2017 / Revised: 25 September 2017

(C) The Author(s) 2017. This article is published with open access at Springerlink.com DOI: $10.1007 / \mathrm{s} 13320-017-0456-7$

Article type: Regular 
linearly dependent on temperature at specific value of temperature [18]. Lee et al. measured the mechanical properties and the elastic properties of monolayer graphene by using nanoindentation in atomic force microscope. They concluded that deformation tests for nanoscale materials could be done outside the linear scale [19]. Wright et al. have observed that graphene has robust nonlinear optical reaction in the terahertz range [20]. Schedin et al. showed that micrometer graphene sensor could detect single actions [21]. The basic functioning idea of graphene sensor depends on the variation of its electrical conductivity because of adsorbed gas molecules on its surface. Bludov et al. investigated the electromagnetic waves travelling on the surface of a nonlinear dielectric material crowned by a graphene sheet [22]. The proposed structure is promising for stability of nonlinear plasmonpolaritons. In addition, dispersion characteristic and sensitivity of different layer waveguide structures including graphene and/or metamaterisls and metallic-plasmonic layer were studied in [23-25].

The focus of this study is on the sensitivity of a waveguide including a thin layer of graphene at the interface between air and dielectric media, which is supported by nonlinear medium. We only consider stabilized nonlinear transverse electric (TE) waves. Furthermore, we are seeking to optimize the sensitivity of the proposed sensor by changing variables such as the waveguide thickness and other physical parameters.

Next section will cover the theoretical background and introduction to the proposed structure. Section 3 will be dedicated to presenting the results and discussion followed by conclusion in Section 4.

\section{Proposed structure and theory}

A three-layer waveguide sensor is presented in Fig. 1. The proposed sensor consists of a graphene thin film laying at the interface between dielectric media and air. The dielectric is placed above a nonlinear media. The graphene is assumed to have conductivity $(\sigma)$ and thickness in the order of $0.142 \mathrm{~nm}$ that will be considered very small and approximated to zero. The dielectric layer has permittivity $\varepsilon_{2}$ and thickness $d$. The nonlinear medium has permittivity expressed by a Kerr-like equation $\varepsilon_{n l}=\varepsilon_{1}+\alpha E_{y}^{2}$, where $\varepsilon_{1}$ is the linear part of the permittivity, $\alpha$ is the strength of the nonlinear part, and $E_{y}$ is the amplitude of the applied filed [11, $12,14]$. Air permittivity $\varepsilon_{3}$ equals to $\varepsilon_{0}$, the vacuum permittivity.

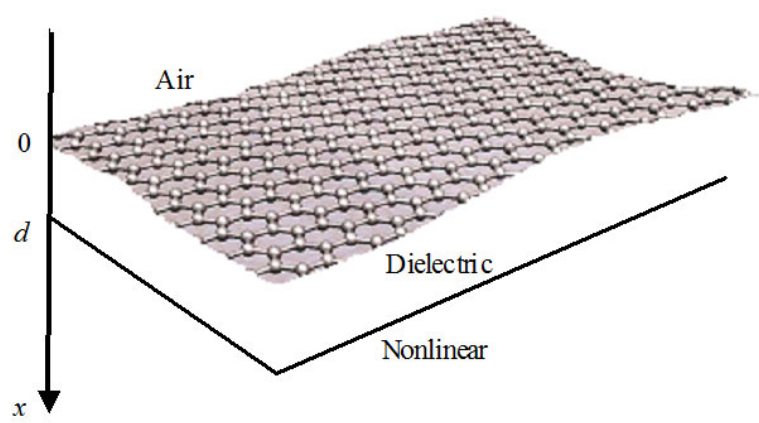

Fig. 1 Proposed structure. The field is assumed to travel along the $z$ axis.

In Fig. 1, the wave is assumed to propagate in the $z$-direction. The electromagnetic field components are $\mathbf{E}=\left\{0, E_{y}, 0\right\}$ and $\mathbf{H}=\left\{H_{x}, 0, H_{z}\right\}$ which can be written in an oscillatory form as

$$
\begin{aligned}
& \mathbf{E}(\mathbf{r}, t)=\mathbf{E}_{y}(x) \mathrm{e}^{\mathrm{i}(\omega t-\beta z)} \\
& \mathbf{H}(\mathbf{r}, t)=\mathbf{H}_{y}(x) \mathrm{e}^{\mathrm{i}(\omega t-\beta z)}
\end{aligned}
$$

where $\omega$ is the wave radian frequency, $\beta=N k_{0}$ is the propagation constant, $N$ is the effective index of refraction, $k_{0}=\omega / c$ is the propagation constant in free space, and $c$ is the speed of light.

Using Maxwell equations and boundary conditions, we obtain the following dispersion equation

$$
\tan \left(\gamma_{2} d-m \pi\right)=\frac{(u+v)}{(1-u v)}
$$

where

$$
\begin{aligned}
& u=\frac{-\gamma_{2}}{\mathrm{i} \sigma \omega-\gamma_{3}}, v=-\frac{\gamma_{2}}{\gamma_{1} \sqrt{1-\frac{k_{0}^{2} r}{\gamma_{1}^{2}}}}, \gamma_{1}=k_{0}^{2} \sqrt{N^{2}-\varepsilon_{1}}, \\
& \gamma_{2}=k_{0}^{2} \sqrt{\varepsilon_{2}-N^{2}}, \quad n_{3}=\sqrt{\varepsilon_{3}}, \text { and } \gamma_{3}=k_{0}^{2} \sqrt{N^{2}-n_{3}^{2}},
\end{aligned}
$$


and $r=\frac{\alpha E^{2}}{2}$ is the nonlinear factor.

The sensitivity $S$ is defined by the variation of the effective refractive index $N$ with respect to the changes in the cladding refractive index $n_{3}$ [9].

$$
S=\frac{\partial N}{\partial n_{3}} \text {. }
$$

To calculate $S$, (3) is differentiated with respect to $n_{3}$. Then, by rearranging the result, we get

$$
S=\frac{\left(Y_{2} X_{2}+Y_{2} v X_{1}\right)}{Z X_{2}^{2}-\left(X_{2} Y_{1}+Y_{3} X_{2}+Y_{1} v X_{1}+X_{1} Y_{3} u\right)}
$$

where

$$
\begin{aligned}
& X_{1}=u+v, X_{2}=1-u v \\
& Y_{1}=\frac{\left(\gamma_{3}\left(\mathrm{i} \sigma \omega-\gamma_{3}\right) k_{0}^{2} N-\gamma_{2}^{2} k_{0}^{2} N\right)}{\gamma_{2} \gamma_{3}\left(\mathrm{i} \sigma \omega-\gamma_{3}\right)^{2}} \\
& Y_{2}=\frac{\gamma_{2} k_{0}^{2} n_{3}}{\gamma_{3}\left(j \sigma \omega-\gamma_{3}\right)^{2}} \\
& Y_{3}=\left(\frac{k_{0}^{2} N}{\gamma_{2} \gamma_{1} \sqrt{1-\frac{k_{0}^{2} r}{\gamma_{1}^{2}}}}+\frac{k_{0}^{2} N \gamma_{2}}{\gamma_{0}^{3} \sqrt{1-\frac{k_{0}^{2} r}{\gamma_{1}^{2}}}}+\frac{k_{0}^{4} N \gamma_{2} r}{\gamma_{0}^{5}\left(1-\frac{k_{0}^{2} r}{\gamma_{1}^{2}}\right)^{1.5}}\right) \\
& Z=-\frac{\sec ^{2}\left(\gamma_{2} d-m \pi\right) k_{0}^{2} N d}{\gamma_{2}} .
\end{aligned}
$$

\section{Results and discussions}

To calculate $S$ for evanescent waves, the dispersion (3) is solved for the effective refractive index $N$, which is plugged into (5). In the calculation, the thickness of conducting graphene layer is assumed infinitesimally thin with constant conductivity $\sigma$.

The sensor layers have a nonmagnetic permeability that is $\mu_{0}=\mu_{2}=\mu_{3}=1$.

The real part of the sensitivity $S$ for $\mathrm{TE}_{0}(m=0)$ is calculated as function of radian frequency $\omega$ at different values of $d$ as displayed in Fig. 2. In the calculation, we consider $\varepsilon_{1}=2.25, \varepsilon_{2}=3, \varepsilon_{3}=1$, $\sigma=6.089 \times 10^{-7}$ Siemens, $r=0.5[26,27]$, and $d$ can take the following values: $75 \mathrm{~nm}, 65 \mathrm{~nm}$, and $55 \mathrm{~nm}$. We realize from Fig. 2 that as the value of $d$ decreases the value of $S$ increases. This can be explained by checking (5). From (5), we notice that $S$ has inverse proportionality with $d$ through the $Z$ term. The $Z$ term for $m=0$ is $-\sec ^{2}\left(\gamma_{2} d\right) k_{0}^{2} N d / \gamma_{2}$. As $d$ increases, the $Z$ term increases, and $S$ decreases. We can also see that as $\omega$ increases the sensitivity increases in case of $d=75 \mathrm{~nm}$ and $d=65 \mathrm{~nm}$. However, in the case of $d=55 \mathrm{~nm}$, it increases slightly, then it decreases.

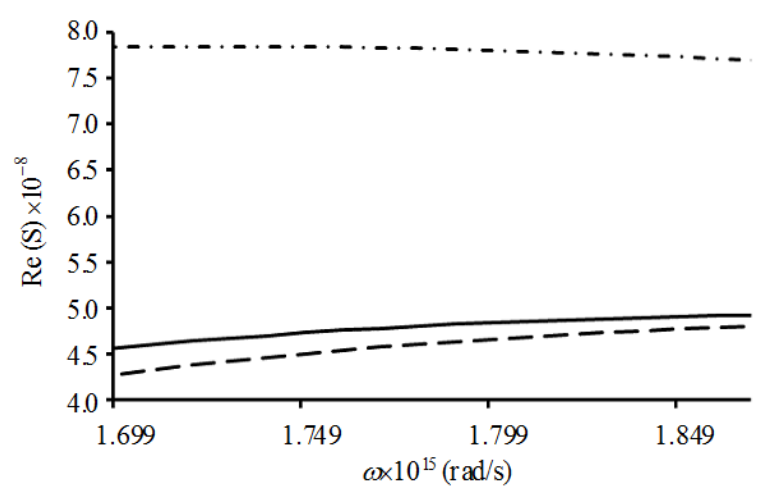

Fig. 2 Real part of the sensitivity for $\mathrm{TE}_{0}$ versus frequency $\sigma=6.089 \times 10^{-7}$ Siemens at different values of $d$. Dash line is when $d=75 \mathrm{~nm}$, solid line is when $d=65 \mathrm{~nm}$, and dash-dotted line is when $d=55 \mathrm{~nm}$.

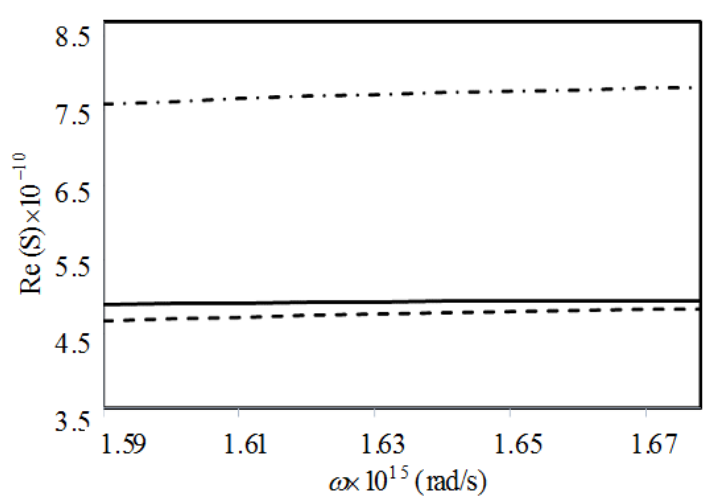

Fig. 3 Real part of the sensitivity for $\mathrm{TE}_{0}$ versus frequency with $\sigma=6.089 \times 10^{-6}$ Siemens at different values of $d$. Dash line is when $d=85 \mathrm{~nm}$, solid line is when $d=75 \mathrm{~nm}$, and dash-dotted line is when $d=55 \mathrm{~nm}$.

To study the effect of the variation of graphene conductivity $\sigma$ on $S$, we repeat the above calculations keeping the same variables and using $\sigma=6.089 \times 10^{-6}$ Siemens. Figure 3 exhibits the real part of the sensitivity $S$ for $\mathrm{TE}_{0}(m=0)$ in terms of radian frequency $\omega$ at different values of $d$. We consider the following values of $d: 85 \mathrm{~nm}, 75 \mathrm{~nm}$, and $55 \mathrm{~nm}$. We obtain similar results as in Fig. 2 that is as $d$ decreases the value of $S$ increases. Also, 
when $\omega$ increases, $S$ increases. However, the values of $S$ decrease by a factor of 100 as $\sigma$ increases by a factor of 10. The same calculations are done at $\sigma=6.089 \times 10^{-5}$ Siemens. Result is displayed in Fig. 4. We obtain similar results with value of $S$ smaller by a factor of 100 .

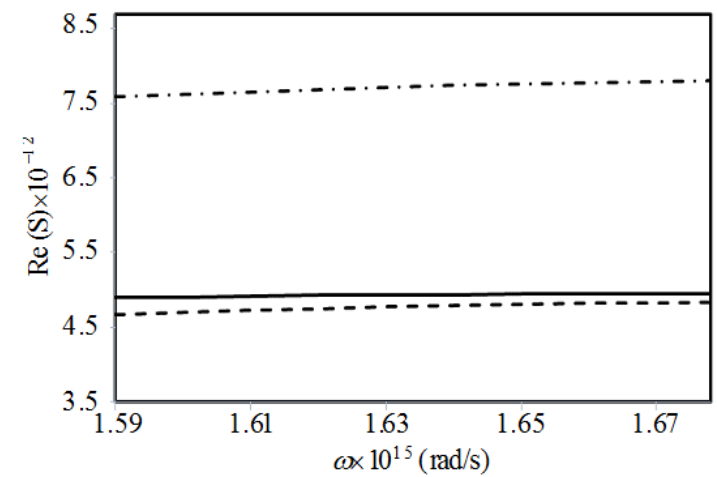

Fig. 4 Real part of the sensitivity for $\mathrm{TE}_{0}$ versus frequency with $\sigma=6.089 \times 10^{-5}$ Siemens at different values of $d$. Dash line is when $d=85 \mathrm{~nm}$, solid line is when $d=75 \mathrm{~nm}$, and dash-dotted line is when $d=55 \mathrm{~nm}$.

The same calculations are performed for $\mathrm{TE}_{1}(m$ $=1$ ). The real part of the sensitivity $S$ is plotted versus frequency $\omega$ at different values of $d$ as shown in Fig. 5. In the calculation, we consider $\varepsilon_{1}=2.25, \varepsilon_{2}=3, \varepsilon_{3}=1, \sigma=6.089 \times 10^{-7}$ Siemens, $r=0.5$, and $d$ takes the following values: $75 \mathrm{~nm}, 65 \mathrm{~nm}$, and $55 \mathrm{~nm}$. We notice from Fig. 5 that as the value of $d$ decreases the value of $S$ increases. In addition, as $\omega$ increases, $S$ increases. Comparing Fig. 2 for $\mathrm{TE}_{0}$ with Fig. 5 for $\mathrm{TE}_{1}$, we conclude that $S$ is smaller for higher mode. That is, $S$ is smaller for $\mathrm{TE}_{1}$ than that for $\mathrm{TE}_{0}$ which can be easily seen form (5).

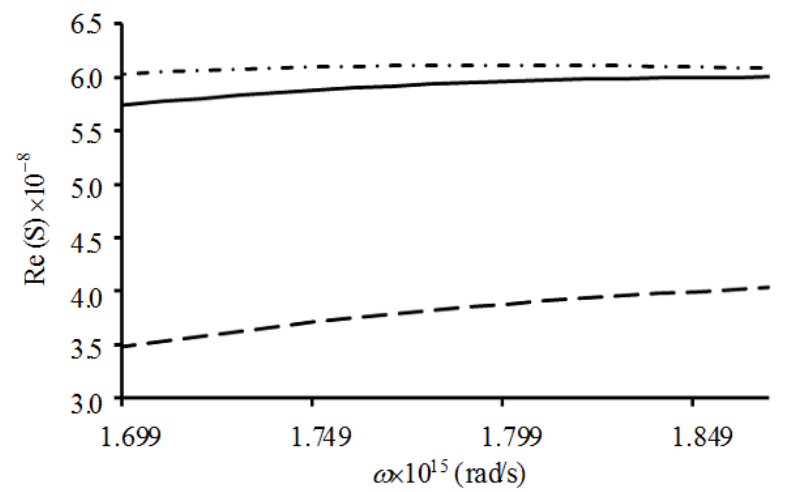

Fig. 5 Real part of the sensitivity for $\mathrm{TE}_{1}$ versus frequency at different values of $d$. Dash line is when $d=75 \mathrm{~nm}$, solid line is when $d=65 \mathrm{~nm}$, and dash-dotted line is when $d=55 \mathrm{~nm}$.

\section{Conclusions}

Three-layer waveguide sensor is proposed in which a graphene thin film with constant conductivity lays at the interface between air and dielectric medium. The dielectric medium is supported by nonlinear media with Kerr-like permittivity. The sensitivity of the sensor is derived from the dispersion equation. The sensitivity has been numerically calculated using Maple as a function of frequency for both $\mathrm{TE}_{0}$ and $\mathrm{TE}_{1}$. Many interesting observations are obtained. The sensitivity is found to decrease for higher modes. It also increases as the thickness of the dielectric decreases. Moreover, the sensitivity decreases as the graphene conductivity increases. Furthermore, it is shown that the sensitivity is directly proportional to the angular frequency. Thus, the sensitivity of the proposed sensor can be controlled by changing the above mentioned variables.

Open Access This article is distributed under the terms of the Creative Commons Attribution 4.0 International License (http://creativecommons.org/ licenses/by/4.0/), which permits unrestricted use, distribution, and reproduction in any medium, provided you give appropriate credit to the original author(s) and the source, provide a link to the Creative Commons license, and indicate if changes were made.

\section{References}

[1] R. A. Potyrailo, S. E. Hobbs, and G. M. Hieftje, "Near-ultraviolet evanescent-wave absorption sensor based on a multimode optical fiber," Analytical Chemistry, 1998, 70(8): 1639-1645.

[2] L. Xu, J. C. Fanuy, K. SSoni, and S. Q. Tao, “Optical fiber humidity sensor based on evanescent-wave scattering," Optics Letters, 2004, 29(11): 1191-1193.

[3] K. A. Remley and A. Weisshaar, "Design and analysis of a silicon-based antiresonant reflecting optical waveguide chemical sensors," Optics Letters, 1996 , 21(16): 1241-1243.

[4] K. Tiefenthaler and W. Lukoz, "Sensitivity of grating couples as integrated optical chemical sensors," Journal of the Optical Society of America $B, 1989,6(2):$ 209-220.

[5] D. K. Qing and I. Yamaguchi, "Analysis of the sensitivity of optical waveguide chemical sensor for 
TM modes by the group-index method," Journal of the Optical Society of America B, 1999, 16(9): 1359-1369.

[6] W. Lukosz, "Integrated optical chemical and direct biochemical sensors," Sensors \& Actuators B: Chemical, 1995, 29(1): 37-50.

[7] R. E. Kunz, "Miniature integrated optical modules for chemical and biochemical sensing," Sensors \& Actuators B: Chemical, 1997, 38(1-3): 13-28.

[8] H. J. El-Khozondar, R. J. El-Khozondar, and S. Zouhdi, "Tunable MTMs consists of a single-walled nanotube thin film waveguide covered by nonlinear cladding," Applied Physics A, 2015, 119(2): 451-453.

[9] H. J. El-Khozondar, M. Müller, R. J. El-Khozondar, M. M. Shabat, and A. W. Koch, "Sensitivity of double-negative metamaterial optical sensor," International Journal of Pure and Applied Sciences and Technology, 2012, 11(2): 29-35.

[10] R. J. El-Khozondar, H. J. El-Khozondar, and M. M. Shabat, "Surface wave propagation in ferroelectric/MTMS interface," Integrated Ferroelectrics, 2011, 130(1): 50-57.

[11] H. J. El-Khozondar, R. J. El-Khozondar, and M. M. Shabat, "Temperature dependence of optical nonlinear waveguide sensor on thermal stress effect," Islamic University Journal for Natural Science and Engineering, 2008, 16(2): 29-40.

[12] H. J. El-Khozondar and R. J. El-Khozondar, "Temperature sensitivity enhancement of nonlinear optical channel waveguide sensors using thermal-stress effect," Islamic University Journal for Natural Science and Engineering, 2008, 16(2): $15-27$.

[13] H. J. El-Khozondar, R. J. El-Khozondar, and M. M. Shabat, "Double-negative metamaterial optical waveguide behavior subjected to stress," Islamic University Journal for Natural Science and Engineering, 2008, 16(1): 9-20.

[14] H. J. El-Khozondar, R. J. El-Khozondar, M. M. Shabat, and A. W. Koch, "Stress effect on optical nonlinear waveguide sensor," Journal of Optical Communications, 2007, 28(3): 175-179.

[15] R. J. El-Khozondar, H. J. El-Khozondar, and M. M. Shabat, "Enhancing sensor sensitivity using graphene-MTM interface," American Journal of Nano Research and Applications, 2017, 4(5): 43-46.

[16] H. J. El-Khozondar, R. J. El-Khozondar, and M. M. Shabat, "Metamaterial-dielectric photonics crystal waveguide structure," Optics, 2015, 4(1-2): 1-4.
[17] K. S. Novoselov, D. Jiang, F. Schedin, T. J. Booth, V. V. Khotkevich, S. V. Morozov, et al., "Two-dimensional atomic crystals," Proceedings of the National Academy of Sciences of USA, 2005, 102(30): 10451-10453.

[18] L. Chen, Z. S. Ma, and C. Zhang, "Vertical absorption edge and temperature dependent resistivity in semihydrogenated graphene," Applied Physics Letters, 2010, 96(2): 023107-1-023107-3.

[19] C. G. Lee, X. D. Wei, J. W. Kysar, and J. Hone, "Measurement of the elastic properties and intrinsic strength of monolayer graphene," Science, 2008, 312(5887): 385-388.

[20] A. R. Wright, X. G. Xu, J. C. Cao, and C. Zhang, "Strong nonlinear optical response of graphene in the terahertz regime," Applied Physics Letters, 2009, 95(7): 072101-1-072101-3.

[21] F. Schedin, A. K. Geim, S. V. Morozov, E. W. Hill, P. Blake, M. I. Katsnelson, et al., "Detection of individual gas molecules adsorbed on graphene," Nature Materials, 2007, 6(9): 652-655.

[22] Y. V. Bludov, D. A. Smirnova, Y. S. Kivshar, N. M. R. Peres, and M. I. Vasilevskiy, "Nonlinear TE-polarized surface polaritons on graphene," Physical Review B, 2014, 89: 035406-1-035406-6.

[23] H. J. El-Khozondar, R. J. El-Khozondar, and M. M. Shabat, "Dispersion characteristics of graphene surface plasmon four layers waveguide," IUG Journal of Natural Studies (IUGNES) Special Issue, 2017, 25(2): 263-266.

[24] H. J. El-Khozondar, R. J. El-Khozondar, and M. M. Shabat, "Dispersion characteristics and sensitivity properties of graphene surface plasmon sensor," Sensor Letters, 2017, 15(3): 249-252.

[25] R. J. El-Khozondar, H. J. El-Khozondar, and M. M. Shabat, "Enhancing sensor sensitivity using graphene-MTM interface," American Journal of Nano Research and Applications, 2016, 4(5): 43-46.

[26] Y. X. Wu, X. Y. Dai, Y. J. Xiang, and D. Y. Fan, "Nonlinear TE-polarized SPPs on a graphene cladded parallel plate waveguide," Journal of Applied Physics, 2017, 121(10): 103103-1103103-7.

[27] Y. Wu, L. Jiang, H. Xu, X. Dai, Y. Xiang, and D. Fan, "Hybrid nonlinear surface-phonon-plasmonpolaritons at the interface of nonlinear medium and graphene-covered hexagonal boron nitride crystal," Optics Express, 2016, 24(3): 2109-2124. 